\title{
INTEGRABILITY OF SUPERHARMONIC FUNCTIONS AND SUBHARMONIC FUNCTIONS
}

\author{
HIROAKI AIKAWA
}

(Communicated by J. Marshall Ash)

Dedicated to Professor Ohtsuka on the occasion of his seventieth birthday

\begin{abstract}
We apply the coarea formula to obtain integrability of superharmonic functions and nonintegrability of subharmonic functions. The results involve the Green function. For a certain domain, say Lipschitz domain, we estimate the Green function and restate the results in terms of the distance from the boundary.
\end{abstract}

\section{INTRODUCTION}

Let $D$ be a domain in $\mathbb{R}^{n}$ with $n \geq 2$. Integrability of superharmonic functions, subharmonic functions and harmonic functions on $D$ has been considered by many authors $[2,3,7-10,12-16]$. In this paper we shall apply the coarea formula to obtain integrability of superharmonic functions and nonintegrability of subharmonic functions. Our results involve the Green function for $D$. Let $D$ be a regular domain with Green function $G(x, y)$. Let $x_{0} \in D$ and write $g(x)=G\left(x, x_{0}\right)$. Our main theorem is the following.

Theorem. Let $\varphi(t)$ be a nonnegative function on $(0, \infty)$. Let $c_{2}=2 \pi$ and $c_{n}=(n-2) \sigma_{n}$ for $n \geq 3$ where $\sigma_{n}$ is the surface measure of a unit sphere.

(i) If $u$ is a superharmonic function on $D$, then

$$
\int_{D} u(x) \varphi(g(x))|\nabla g(x)|^{2} d x \leq c_{n} u\left(x_{0}\right) \int_{0}^{\infty} \varphi(t) d t .
$$

(ii) If $s$ is a subharmonic function on $D$, then

$$
\int_{D} s(x) \varphi(g(x))|\nabla g(x)|^{2} d x \geq c_{n} s\left(x_{0}\right) \int_{0}^{\infty} \varphi(t) d t .
$$

Our Theorem has the following corollaries. Let $D$ be a proper subdomain and put $\delta(x)=\operatorname{dist}(x, \partial D)$. Since $g(x)$ is a positive harmonic function on $D \backslash\left\{x_{0}\right\}$, it is easy to see that $|\nabla g(x)| \leq M g(x) / \delta(x)$ for $x$ apart from a

Received by the editors January 6, 1992.

1991 Mathematics Subject Classification. Primary 31B05.

Key words and phrases. Superharmonic function, subharmonic function, Green function, coarea formula.

(C) 1993 American Mathematical Society $0002-9939 / 93 \$ 1.00+\$ .25$ per page 
neighborhocd of $x_{0}$. Let $x_{1} \in D$. By the Harnack principle there is a constant $M>1$ such that $M^{-1} g(x) \leq G\left(x, x_{1}\right) \leq M g(x)$ for $x$ close to the boundary. Hence we have

Corollary 1. Let $\varphi$ satisfy the doubling condition:

$$
\sup _{T \leq t \leq 2 T} \varphi(t) \leq M \inf _{T \leq t \leq 2 T} \varphi(t)
$$

for $T>0$ with $M \geq 1$ independent of $T$. Suppose

$$
\int_{0}^{T} \varphi(t) d t=\infty \text { for some } T>0 .
$$

If $s$ is a nonnegative nonzero subharmonic function on $D$, then for any compact subset $E$ of $D$

$$
\int_{D \backslash E} s(x) \varphi(g(x)) \frac{g(x)^{2}}{\delta(x)^{2}} d x=\infty .
$$

For some domains we can estimate $g(x)$ by $\delta(x)$. By $B(x, r)$ we denote the open ball with radius $r$ and center at $x$. We say that $D$ is uniformly $\Delta$-regular if there is a constant $\varepsilon_{1}, 0<\varepsilon_{1}<1$, such that, for all $x \in \partial D$ and all $r, 0<r<r_{0}$,

$$
w_{x, r} \leq 1-\varepsilon_{1} \quad \text { on } B(x, r / 2) \cap D,
$$

where $w_{x, r}$ is the harmonic measure of $\partial B(x, r) \cap D$ in the region $B(x, r) \cap D$ [1, Definition 2]. It is known that $D$ is uniformly $\Delta$-regular if and only if it satisfies the capacity density condition (CDC) (see $[6,18])$. For such a domain there are constants $\beta, 0<\beta \leq 1$, and $M>0$ such that

$$
g(x) \leq M \delta(x)^{\beta}
$$

for $x$ close to $\partial D$ (see [1]). Letting $\varphi(t)=1 / t$ in Corollary 1, we obtain

Corollary 2. Let $D$ be a uniformly $\Delta$-regular domain and let $\beta$ be as above. If $s$ is a nonnegative nonzero subharmonic function on $D$, then for any compact subset $E$ of $D$

$$
\int_{D \backslash E} s(x) \delta(x)^{\beta-2} d x=\infty .
$$

Let $T_{\psi}=\left\{x: x_{n}>|x| \cos \psi\right\}$. This is a cone with vertex at the origin and aperture $\psi$. It is not so difficult to see that there is a positive harmonic function $u_{\psi}$ on $T_{\psi}$ such that $u_{\psi}=0$ on $\partial T_{\psi}$; such a function $u_{\psi}$ is unique up to a multiplicative constant and is homogeneous of degree $\alpha=\alpha_{n}(\psi)>0$, i.e., $u_{\psi}(r x)=r^{\alpha} u_{\psi}(x)$ for $r>0$. This constant $\alpha_{n}(\psi)$ is referred to as the maximal order of barriers (see [7, p. 271]). It is known that $\alpha_{n}$ is strictly decreasing; $\alpha_{n}(\pi / 2)=1 ; \lim _{\psi \rightarrow 0} \alpha_{n}(\psi)=\infty ; \lim _{\psi \rightarrow \pi} \alpha_{n}(\psi)=0$ (for $n \geq 3$ ); $\alpha_{2}(\psi)=\pi /(2 \psi) ;$ and $\alpha_{4}(\psi)=\pi / \psi-1$.

We say that $D$ satisfies the exterior cone condition with aperture $\psi$ if there exists $\rho>0$ such that for each $y \in \partial D$ there is a truncated cone of radius $\rho$ with vertex at $y$ and aperture $\psi$ lying outside $D$. It is easy to see that if $D$ satisfies the exterior cone condition with aperture $\psi$, then $D$ is uniformly $\Delta$-regular and the constant $\beta$ in (4) can be taken as $\alpha_{n}(\pi-\psi)$. Estimating the derivative of a certain conformal mapping, Masumoto [8] proved this result for the plane case. (Note that the constant $\theta$ in his notation is related to $\psi$ as $\pi \theta=2 \psi$.) 
Corollary 3. Let $D$ satisfy the exterior cone condition with aperture $\psi$ and let $\beta=\alpha_{n}(\pi-\psi)$. If $s$ is a nonnegative nonzero subharmonic function on $D$, then (5) holds for any compact subset $E$ of $D$.

Our Theorem also yields integrability of superharmonic functions. For this purpose we need to give a lower bound of $|\nabla g(x)|$. It is, in general, difficult to obtain the bound; the pointwise estimate $|\nabla g(x)| \geq M g(x) / \delta(x)$ does not hold. However, we can prove that $|\nabla g(x)| \geq M g(x) / \delta(x)$ in a certain sense (see Lemma 1 below) for an NTA domain introduced by [5]. An NTA domain is uniformly $\Delta$-regular. As a result we have

Corollary 4. Let $D$ be an NTA domain. Let $\varphi$ satisfy (1). Suppose

$$
\int_{0}^{T} \varphi(t) d t<\infty \text { for } T>0 .
$$

Then every nonnegative superharmonic function $u$ on $D$ satisfies

$$
\int_{D \backslash B\left(x_{0}, r_{1}\right)} u(x) \varphi(g(x)) \frac{g(x)^{2}}{\delta(x)^{2}} d x<\infty
$$

for any $r_{1}>0$.

We say that a bounded domain $D$ is $k$-Lipschitz if $D$ and $\partial D$ are given locally by a Lipschitz function whose Lipschitz constant is at most $k$. If $D$ is $k$-Lipschitz for some $k>0$, then we say that $D$ is a Lipschitz domain. A Lipschitz domain is an NTA domain. Let $D$ be a $k$-Lipschitz domain and let $\alpha_{n}$ be the maximal order of barriers as before. Then it is known that

$$
\begin{array}{ll}
g(x) \geq M \delta(x)^{\alpha} & \text { for } x \in D, \\
g(x) \leq M \delta(x)^{\beta} & \text { for } x \in D \backslash B\left(x_{0}, r_{2}\right),
\end{array}
$$

where $\alpha=\alpha_{n}(\psi), \beta=\alpha_{n}(\pi-\psi)$, and $\psi=\arctan (1 / k), 0<\psi<\pi / 2[7$, Proposition 2]. We remark that $0<\beta<1<\alpha$. Since $\int_{0} t^{\varepsilon-1} d t<\infty$ for $\varepsilon>0$, we obtain the following corollary, which improves [7, Theorem 8] with $p=1$.

Corollary 5. Let $D$ be a $k$-Lipschitz domain and let $\alpha$ and $\beta$ be as above.

(i) If $u$ is a nonnegative superharmonic function on $D$, then for $\varepsilon>0$

$$
\int_{D} u(x) \delta(x)^{\varepsilon+\alpha-2} d x<\infty .
$$

(ii) If $s$ is a nonnegative nonzero subharmonic function on $D$, then for any compact subset $E$ of $D$

$$
\int_{D \backslash E} s(x) \delta(x)^{\beta-2} d x=\infty .
$$

For the plane case Masumoto [9] proved a result more general than the above (i). He also informed us that Stegenga and Ullrich [13] recently proved the integrability of superharmonic functions on a Hölder domain and a John domain. However, our method is completely different and gives sharp exponents for at least Lipschitz domains (see also Corollary 6 below). Another advantage of 
the use of the coarea formula is that it enables us to deal with superharmonic functions and subharmonic functions, simultaneously.

Recently, Maeda pointed out that $\alpha_{n}(\psi)=2$ for $\cos \psi=1 / \sqrt{n}$. Hence we have the following: If $0<k<1 / \sqrt{n-1}$, then every nonnegative superharmonic function on a $k$-Lipschitz domain $D$ is integrable over $D$.

The plan of this paper is as follows. We prove our Theorem in the next section, and Corollary 4 in $\S 3$. Other corollaries are almost straightforward. In $\S 4$ we give some $L^{p}$-integrability results.

I would like to thank Professors Fumi-Yuki Maeda, Makoto Masumoto, and Noriaki Suzuki for valuable conversations.

\section{Proof of The Theorem}

Our main tool for the proof of the Theorem is the coarea formula. For the reader's convenience we state it below. For a proof see, e.g., [11, pp. 37-39].

Lemma (Coarea formula). Let $\Omega$ be an open set in $\mathbb{R}^{n}$. Suppose $\psi$ is a Borel measurable function on $\Omega$ and $f$ is a smooth function on $\Omega$. Then

$$
\int_{\Omega} \psi(x)|\nabla f(x)| d x=\int_{0}^{\infty} d t \int_{\{x \in \Omega:|f(x)|=t\}} \psi(x) d \sigma(x),
$$

where $\sigma$ is the surface measure.

Proof of Theorem. Let $D_{t}=\{x \in D: g(x)>t\}$ and let $G_{t}(x, y)$ be the Green function for $D_{t}$. Obviously $x_{0} \in D_{t}$ and $D_{t} \uparrow D$ as $t \downarrow 0$. By the Sard theorem (see, e.g., [11, Corollary, p. 35]), we find a set $\mathscr{E}$ of linear measure 0 in $(0, \infty)$ such that $\partial D_{t}=\{x \in D: g(x)=t\}$ is smooth for $t \notin \mathscr{E}$. Observe that $G_{t}\left(x, x_{0}\right)=g(x)-t$, and hence the harmonic measure $\omega_{t}$ at $x_{0}$ for $D_{t}$ is given by

$$
c_{n} \frac{d \omega_{t}}{d \sigma}=-\frac{\partial G_{t}\left(\cdot, x_{0}\right)}{\partial n}=-\frac{\partial g}{\partial n}=|\nabla g| \quad \text { on } \partial D_{t}
$$

for $t \notin \mathscr{E}$, since the outward normal $n$ on $\partial D_{t}$ is equal to $-\nabla g /|\nabla g|$. Hence the Poisson integral formula becomes

$$
\begin{aligned}
& u\left(x_{0}\right) \geq \frac{1}{c_{n}} \int_{\partial D_{t}} u|\nabla g| d \sigma \text { for a superharmonic function } u \text { on } D, \\
& s\left(x_{0}\right) \leq \frac{1}{c_{n}} \int_{\partial D_{t}} s|\nabla g| d \sigma \quad \text { for a subharmonic function } s \text { on } D .
\end{aligned}
$$

Let us invoke the coarea formula with $\psi=u \varphi(g)|\nabla g|, f=g$, and $\Omega=D$. Then

$$
\int_{D} u(x) \varphi(g(x))|\nabla g(x)|^{2} d x=\int_{0}^{\infty} \varphi(t) d t \int_{\partial D_{t}} u|\nabla g| d \sigma \leq c_{n} u\left(x_{0}\right) \int_{0}^{\infty} \varphi(t) d t .
$$

Thus (i) follows. Similarly, we obtain (ii) by letting $\psi=s \varphi(g)|\nabla g|, f=g$, and $\Omega=D$. The Theorem is proved.

Remark. Letting $\varphi(t)=1 / T$ for $0<t<T$, we obtain the following:

(i) If $u$ is a superharmonic function on $D$, then

$$
u\left(x_{0}\right) \geq \frac{1}{c_{n}} \limsup _{T \rightarrow 0} \frac{1}{T} \int_{\{x \in D: 0<g(x)<T\}} u(x)|\nabla g(x)|^{2} d x .
$$


(ii) If $s$ is a subharmonic function on $D$, then

$$
s\left(x_{0}\right) \leq \frac{1}{c_{n}} \liminf _{T \rightarrow 0} \frac{1}{T} \int_{\{x \in D: 0<g(x)<T\}} s(x)|\nabla g(x)|^{2} d x .
$$

\section{Proof of Corollary 4}

Let $\left\{Q_{j}\right\}$ be the Whitney decomposition of $D$. We may assume that $x_{0} \in Q_{0}$ and we shall omit this cube $Q_{0}$ in the succeeding argument. For each Whitney cube $Q_{j}$ we let $x_{j}$ be the center of $Q_{j}, r_{j}$ the diameter of $Q_{j}$, and $t_{j}=$ $\operatorname{dist}\left(Q_{j}, \partial D\right)$. We write $M$ for a positive constant independent of $Q_{j}$, whose value may change from one occurrence to the next. If $M^{-1} f \leq g \leq M f$ for two positive quantities $f$ and $g$, then we write $f \approx g$. Observe that $r_{j} \approx t_{j}$. Let $x_{j}^{*} \in \partial D$ be a point such that $\operatorname{dist}\left(x_{j}^{*}, Q_{j}\right)=t_{j}$. It is well known that the boundary Harnack principle holds for an NTA domain [5, Lemma 4.10]. In view of (3) and the boundary Harnack principle, we can find a constant $M_{1}>1$ such that

$$
g(x) \leq\left(1-\varepsilon_{1}\right) \inf _{Q_{j}} g \text { for } x \in D \cap B\left(x_{j}^{*}, t_{j} / M_{1}\right) .
$$

Hence we can find a constant $M_{2}$ with the following property: Let $\widetilde{Q}_{j}$ be the cube with the same center $x_{j}$ as $Q_{j}$ but expanded $M_{2}$ times. Then

(i) there is a point $x^{\prime} \in \widetilde{Q}_{j}$ such that $g\left(x^{\prime}\right) \leq\left(1-\varepsilon_{1}\right) \inf _{Q_{j}} g$;

(ii) $\operatorname{dist}\left(\widetilde{Q}_{j}, \partial D\right) \approx r_{j}$.

Observe that only a finite number of cubes $\widetilde{Q}_{0}, \ldots, \widetilde{Q}_{j_{0}}$ meet $\widetilde{Q}_{0}$. We shall omit these cubes in the succeeding argument. Let $g\left(x_{j}\right)=g_{j}$. By the Harnack inequality $g(x) \approx g_{j}$ for $x \in Q_{j}$. By $m(E)$ we denote the Lebesgue measure of $E$.

Lemma 1. Let $\widetilde{Q}_{j}$ be as above. There exists a positive constant $\varepsilon_{2}$ such that

$$
E_{j}=\left\{x \in \widetilde{Q}_{j}:|\nabla g(x)| \geq \varepsilon_{2} g_{j} / r_{j}\right\}
$$

satisfies $m\left(E_{j}\right) \geq M m\left(\widetilde{Q}_{j}\right)$.

Proof. Observe that $|\nabla g| \leq M g_{j} / r_{j}$ on $\tilde{Q}_{j}$. Let $x^{\prime} \in \tilde{Q}_{j}$ be as before the lemma. For $x \in Q_{j}$ we let $l$ be the line segment connecting $x^{\prime}$ and $x$. Note that $l \subset \widetilde{Q}_{j}$ and $|l| \leq M r_{j}$ uniformly for $x \in Q_{j}$, where $|l|$ is the length of $l$. We have for $\varepsilon>0$

$$
\begin{aligned}
\varepsilon_{1} g_{j} & \leq M \varepsilon_{1} g(x) \leq M\left(g(x)-g\left(x^{\prime}\right)\right) \leq M \int_{l}|\nabla g| d s \\
& \leq M \int_{\left\{y \in l:|\nabla g(y)| \leq \varepsilon g_{j} / r_{j}\right\}} \varepsilon \frac{g_{j}}{r_{j}} d s+M \int_{\left\{y \in l:|\nabla g(y)| \geq \varepsilon g_{j} / r_{j}\right\}} M \frac{g_{j}}{r_{j}} d s \\
& \leq M|l| \varepsilon \frac{g_{j}}{r_{j}}+M \frac{g_{j}}{r_{j}}\left|\left\{y \in l:|\nabla g(y)| \geq \varepsilon \frac{g_{j}}{r_{j}}\right\}\right| \\
& \leq M 3 \varepsilon g_{j}+M \frac{g_{j}}{r_{j}}\left|\left\{y \in l:|\nabla g(y)| \geq \varepsilon \frac{g_{j}}{r_{j}}\right\}\right| .
\end{aligned}
$$


Letting $\varepsilon>0$ so small that $M_{3} \varepsilon \leq \varepsilon_{1} / 2$, we obtain

$$
\frac{\varepsilon_{1}}{2} g_{j} \leq M \frac{g_{j}}{r_{j}}\left|\left\{y \in l:|\nabla g(y)| \geq \varepsilon \frac{g_{j}}{r_{j}}\right\}\right|,
$$

whence $\left|\left\{y \in l:|\nabla g(y)| \geq \varepsilon g_{j} / r_{j}\right\}\right| \geq M r_{j}$. This inequality holds for any $x \in$ $Q_{j}$. Therefore, Fubini's theorem asserts that for $\varepsilon_{2}=\varepsilon$

$$
E_{j}=\left\{y \in \widetilde{Q}_{j}:|\nabla g(y)| \geq \varepsilon_{2} g_{j} / r_{j}\right\}
$$

satisfies $m\left(E_{j}\right) \geq M m\left(\widetilde{Q}_{j}\right)$.

Lemma 2. Let $Q$ be a cube in $\mathbb{R}^{n}$. For $M_{4}>1$ we let $Q^{*}$ be the cube with the same center as $Q$ but expanded $M_{4}$ times. Suppose $E$ is a measurable subset of $Q$. If $u$ is a nonnegative superharmonic function on $Q^{*}$, then

$$
\frac{1}{m(Q)} \int_{Q} u d x \leq \frac{M_{5}}{m(E)} \int_{E} u d x,
$$

where $M_{5}$ depends only on the dimension and $M_{4}$.

Proof. Let $G^{*}(x, y)$ be the Green function for $Q^{*}$. Taking the balayage over $Q$, if necessary, we may assume that $u$ is a Green potential $\int G^{*}(\cdot, y) d \mu(y)$ with measure $\mu$ on the closure of $Q$. Let $r$ be the diameter of $Q$. Then it is easy to see that

$$
M r^{2-n} m(E) \leq \int_{E} G^{*}(x, y) d x \leq \int_{Q} G^{*}(x, y) d x \leq M r^{2}
$$

uniformly for $y$ in the closure of $Q$, where $M$ depends only on the dimension and $M_{4}$. Hence Fubini's theorem yields

$$
\frac{1}{m(Q)} \int_{Q} u d x \leq \frac{M r^{2}}{m(Q)}\|\mu\|=M r^{2-n}\|\mu\| \leq \frac{M}{m(E)} \int_{E} u d x .
$$

The lemma is proved.

Proof of Corollary 4. By (1) and the Harnack inequality we have

$$
\varphi(g(x)) g(x)^{2} \delta(x)^{-2} \approx \varphi\left(g_{j}\right) g_{j}^{2} r_{j}^{-2}
$$

for $x \in \widetilde{Q}_{j}$. Let us apply Lemma 2 to $Q=\widetilde{Q}_{j}$ and $E=E_{j}$. Then we obtain from Lemma 1 that

$$
\begin{aligned}
\int_{\widetilde{Q}_{j}} u \varphi(g) g^{2} \delta^{-2} d x & \leq M \varphi\left(g_{j}\right) g_{j}^{2} r_{j}^{-2} \int_{\widetilde{Q}_{j}} u d x \leq M \varphi\left(g_{j}\right) g_{j}^{2} r_{j}^{-2} \int_{E_{j}} u d x \\
& \leq M \int_{E_{j}} u \varphi\left(g_{j}\right)|\nabla g|^{2} d x \leq M \int_{\widetilde{Q}_{j}} u \varphi(g)|\nabla g|^{2} d x .
\end{aligned}
$$

Note that the multiplicity of $\widetilde{Q}_{j}$ is bounded, i.e., $\sum \chi_{\widetilde{Q}_{j}} \leq N$. Summing up the integral over $\widetilde{Q}_{j}$, we obtain from the Theorem that

$$
\int_{D \backslash\left(\widetilde{Q}_{0} \cup \cdots \cup \widetilde{Q}_{J_{0}}\right)} u \varphi(g) g^{2} \delta^{-2} d x \leq M \int_{D \backslash \widetilde{Q}_{0}} u \varphi(g)|\nabla g|^{2} d x \leq M u\left(x_{0}\right)<\infty .
$$

Since $u \varphi(g) g^{2} \delta^{-2}$ is integrable on any compact subset of $D \backslash\left\{x_{0}\right\}$, we obtain the corollary. 


\section{4. $L^{p}$-INTEGRABILITY}

In this section we shall prove the following corollary, which gives an answer to Problems 3.32 and 3.34 raised by Armitage and Gardiner in [4].

Corollary 6. Let $D$ be a $k$-Lipschitz domain and let $\alpha$ and $\beta$ be as in Corollary 5.

(i) If $u$ is a nonnegative superharmonic function on $D$, then

$$
\int_{D} u(x)^{p} d x<\infty
$$

for $0<p<\min \{n /(n+\alpha-2), 1 /(\alpha-1)\}$.

(ii) Let $0<p \leq 1$. If $s$ is a nonnegative nonzero subharmonic function on $D$, then for any compact subset $E$ of $D$

$$
\int_{D \backslash E} \frac{s(x)^{p}}{\delta(x)^{n-n p+(2-\beta) p}} d x=\infty .
$$

We remark that $0<\beta<1<\alpha$ and

$$
\min \{n /(n+\alpha-2), 1 /(\alpha-1)\}= \begin{cases}n /(n+\alpha-2) & \text { if } 1<\alpha \leq 2, \\ 1 /(\alpha-1) & \text { if } \alpha>2\end{cases}
$$

If $s$ is a nonnegative subharmonic function, then so is $s^{p}$ for $p>1$. Thus the case $p>1$ is irrelevant for nonintegrability of subharmonic functions. It is easy to see that the bound of $p$ in Corollary 6 is sharp. In particular, the bound given in [7] is improved. For the plane case Masumoto [8, 9] gave the same bound of $p$. Observe that if $D$ is a bounded Lipschitz domain, then for $\varepsilon<1$

$$
\int_{D} \delta(x)^{-\varepsilon} d x<\infty
$$

In view of Corollary 5 , we obtain that Corollary 6(i) follows from the next lemma.

Lemma 3. Let $D$ be a bounded domain and suppose that $u$ is a nonnegative superharmonic function on $D$.

(i) If $0 \leq \gamma<1$ and $0<p \leq n /(n-\gamma)$, then

$$
\left(\int_{D} u(x)^{p} d x\right)^{1 / p} \leq M \int_{D} u(x) \delta(x)^{-\gamma} d x
$$

(ii) Suppose (6) holds for some $\varepsilon>0$. If $\gamma<0$ and $0<p<\varepsilon /(\varepsilon-\gamma)$, then (7) holds.

Proof. For (i) we may assume that $p \geq 1$. Let $\left\{Q_{j}\right\}$ be the Whitney decomposition of $D$. By an argument similar to Lemma 2 we have

$$
\left(\int_{Q_{j}} u(x)^{p} d x\right)^{1 / p} \leq M(\operatorname{diam} D)^{\gamma-n+p / n} \int_{Q_{j}} u(x) \delta(x)^{-\gamma} d x
$$

since $\gamma-n+p / n \geq 0$ by assumption. Summing up the above integral, we obtain (7). 
We note that $p<\varepsilon /(\varepsilon-\gamma)<1$ for (ii). Using the Hölder inequality with $1 / p>1$, we obtain

$$
\int_{D} u(x)^{p} d x \leq\left(\int_{D} u(x) \delta(x)^{-\gamma} d x\right)^{p}\left(\int_{D} \delta(x)^{p \gamma /(1-p)} d x\right)^{1-p} .
$$

Since $0<p<\varepsilon /(\varepsilon-\gamma)$ implies $p \gamma /(1-p)>-\varepsilon$, we obtain (7). The lemma is proved.

For the proof of Corollary 6(ii), it is sufficient to show the following lemma.

Lemma 4. Let $\varphi$ satisfy (1) and let $0<p<1$. Suppose $s$ is a nonnegative subharmonic function on $D$. Then

$$
\begin{aligned}
& \int_{D \backslash B\left(x_{0}, r\right)} s(x) \varphi(g(x)) \frac{g(x)^{2}}{\delta(x)^{2}} d x \\
& \leq M\left(\int_{D \backslash B\left(x_{0}, r\right)} \frac{s(x)^{p} \varphi(g(x))^{p} g(x)^{2 p}}{\delta(x)^{n-n p+2 p}} d x\right)^{1 / p}
\end{aligned}
$$

for $0<r<\delta\left(x_{0}\right) / 2$.

Proof. We denote by $I$ the integral in the right-hand side of (8). Put $k=$ $r /\left(2 \delta\left(x_{0}\right)\right)$. As observed in [14, Proof of Theorem 2], we have

$$
s(x)^{p} \leq M \delta(x)^{-n} \int_{B(x, k \delta(x))} s(y)^{p} d y,
$$

where $M$ depends only on $p, k$, and the dimension. For $x \in D \backslash B\left(x_{0}, r\right)$ we have

$$
\delta(x) \leq\left|x-x_{0}\right|+\delta\left(x_{0}\right) \leq\left(1+\frac{\delta\left(x_{0}\right)}{r}\right)\left|x-x_{0}\right|<\frac{3}{4 k}\left|x-x_{0}\right|,
$$

so that $g=G\left(\cdot, x_{0}\right)$ is harmonic on $B\left(x, \frac{4}{3} k \delta(x)\right)$. Hence (1), (9), and the Harnack inequality yield

$$
\begin{aligned}
s(x)^{p} & \leq M \delta(x)^{(2-n) p} \varphi(g(x))^{-p} g(x)^{-2 p} \int_{B(x, k \delta(x))} \frac{s(y)^{p} \varphi(g(y))^{p} g(y)^{2 p}}{\delta(y)^{n-n p+2 p}} d y \\
& \leq M \delta(x)^{(2-n) p} \varphi(g(x))^{-p} g(x)^{-2 p} I .
\end{aligned}
$$

Therefore

$$
s(x)=s(x)^{p} \cdot s(x)^{1-p} \leq M s(x)^{p}\left(\delta(x)^{(2-n) p} \varphi(g(x))^{-p} g(x)^{-2 p} I\right)^{(1-p) / p} .
$$

Substituting this inequality in the left-hand side of (8), we obtain the lemma.

\section{NOTE ADDED IN PROOF}

Professor Peter Lindqvist informed me that he proved the integrability of a positive supersolution of certain nonlinear equations, such as the $p$-Laplace equation (J. Analyse Math. 62 (to appear)). He used the BMO-norm estimate and an argument similar to [12, 13]. 


\section{REFERENCES}

1. A. Ancona, On strong barriers and an inequality of Hardy for domains in $\mathbb{R}^{n}$, J. London Math. Soc. (2) 34 (1986), 274-290.

2. D. H. Armitage, On the global integrability of superharmonic functions in balls, J. London Math. Soc. (2) 4 (1971), 365-373.

3. Soc. (2) 6 (1972), 109-121.

4. D. A. Brannan and W. K. Hayman, Research problems in complex analysis, Bull. London Math. Soc. 21 (1989), 1-35.

5. D. S. Jerison and C. E. Kenig, Boundary behavior of harmonic functions in non-tangentially accessible domains, Adv. in Math. 46 (1982), 80-147.

6. P. Jones and T. Wolff, Hausdorff dimension of harmonic measures in the plane, Acta Math. 161 (1988), 131-144.

7. F.-Y. Maeda and N. Suzuki, The integrability of superharmonic functions on Lipschitz domains, Bull. London Math. Soc. 21 (1989), 270-278.

8. M. Masumoto, $A$ distorsion theorem for conformal mappings with an application to subharmonic functions, Hiroshima Math. J. 20 (1990), 341-350.

9. __ Integrability of superharmonic functions on plane domains, J. London Math. Soc. (2) 45 (1992), 62-78.

10. Integrability of superharmonic functions on Hölder domains of the plane, $\mathrm{P}_{\mathbf{i}}$, In. Amer. Math. Soc. 117 (1993), 1083-1088.

11. V. G. Maz'ya, Sobolev spaces, Springer, New York, 1985.

12. W. Smith and D. A. Stegenga, Sobolev imbedding and integrability of harmonic functions on Hölder domains, Potential Theory (Proc. Internat. Conf. Potential Theory, Nagoya 1990) (M. Kishi, ed.), Walter de Gruyter, Berlin, 1992, pp. 303-313.

13. D. A. Stegenga and D. C. Ullrich, Superharmonic functions in Hölder domains, preprint.

14. N. Suzuki, Nonintegrability of harmonic functions in a domain, Japan. J. Math. 16 (1990), 269-278.

15. _ Nonintegrability of superharmonic functions, Proc. Amer. Math. Soc. 113 (1991), 113-115.

16. - Note on the integrability of superharmonic functions, preprint.

17. K.-O. Widman, Inequalities for the Green function and boundary continuity of the gradient of solutions of elliptic differential equations, Math. Scand. 21 (1967), 17-37.

18. J.-M. Wu, Boundary density and the Green function, Michigan Math. J. 38 (1991), 175-189.

Department of Mathematics, Gunma University, Kiryu 376, Japan

Current address: Department of Mathematics, Faculty of Science, Kumamoto University, Kumamoto 860, Japan

E-mail address: aikawa@kumamoto-u.ac.jp 